\title{
Orthostatic hypoxia after device closure of ruptured sinus Valsalva: Platypnea-orthodeoxia syndrome
}

\author{
Yaser Jenab ${ }^{1}$, ali hosseinsabet ${ }^{2}$, sara taghi ${ }^{2}$, kaveh hosseini ${ }^{2}$, and laura vaskelyte ${ }^{2}$ \\ ${ }^{1}$ Tehran University of Medical Sciences \\ ${ }^{2}$ Affiliation not available
}

September 22, 2020

\begin{abstract}
A fifty years old woman with dyspnea of exertion and rupture of sinus Valsalva to right atrium in echocardiography referred

to our hospital. After device closure of the rupture, she developed orthostatic hypoxemia. Invasive intra-cardiac evaluation confirmed the diagnosis of Platypnea-Orthodeoxia Syndrome which was managed by device closure for PFO.
\end{abstract}

\section{Introduction}

Rupture of sinus Valsalva (RSVA) to right atrium(RA) causes significant left to right shunt, tricuspid regurgitation, RV failure and finally biventricular heart failure may happen[1]. If being uncorrected it will result in deterioration of heart function, hence, early invasive management is advised. If possible, trans-catheter approach is preferred over open surgery in cases with RSVA. Coexisting cardiac malformation may be present in patients with SVA. Ventricular septal defect or aortic valve regurgitation may be present in about 30 to $40 \%$ of patients[2]. Recent report by Mittal et al, described a case with newly diagnosed un-ruptured sinus Valsalva and previous history of ASD closure during infancy[3]. Platypnea-orthodeoxia (POS) is also a rare condition in which the patient becomes hypoxic in the upright position. Herein we presented a case with POS after RSVA device closure.

\section{Case presentation}

Fifty years old woman with chief complaint of dyspnea of exertion functional class III from 8 months ago and suspicious intra-cardiac shunt referred to Tehran Heart Center, a referral educational hospital in Tehran, Iran, for further evaluation. Ehocardiography showed normal left ventricle function (Ejection Fraction 55\%), mildly dilated right ventricle (RV) and moderate tricuspid regurgitation (TR). An important finding was diastolic turbulent flow from right coronary cusp (RCC) of aortic valve to right atrium (RA) at the level of tricuspid valve septal leaflet, suggestive for rupture of sinus Valsalva.

At the time of admission her physical exams were as follows: blood pressure 128/68 $\mathrm{mmHg}$, heart rate 93 bpm and respiratory rate 19 . Her O2 saturation in ambient air was $94 \%$ with no sign of peripheral or central cyanosis. In heart \& lung examination, continuous murmur was auscultated simply in left parasternal border. No rales or crackle was heard.

Past medical history was unremarkable except hypertension and recent COVID-19. COVID-19 was diagnosed 3 months ago and was treated in home with supportive therapy.

Based on these findings she was planned to undergo transesophageal echocardiography (TEE) and cardiac catheterization in order to decide the possibility of percutaneous closure of RCC to RA rupture. In-cath lab TEE showed ruptured RCC with continuous flow shunt to RA (Fig. 1; A). The aortic and RA orifices were 8 and $6 \mathrm{~mm}$, respectively. The distance between aortic valve to right coronary artery (RCA) was $12 \mathrm{~mm}$. 
Patent foramen oval (PFO) was also visible. Cardiac catheterization showed significant flow from aorta to RA in aortic root injection (Fig. 1; B). The Qp/Qs ratio was 2.3 in favor of significant left to right shunt (aorta to RA). She was planned for device closure at the next session with patent ductus arteriosus (PDA) device.

After aortic root injection, wiring (0.035 wire) was done through sinus rupture to pulmonary artery (PA) and was snared and externalized via right femoral vein. Delivery sheath was inserted through rupture and Occlutech PDA occluder 12-15 was deployed to close the rupture (Fig 1; C). Afterwards, aortic root injection showed mild residual shunt and TEE confirmed it.

The patient was transferred to the critical care unit (CCU) and monitored closely. However, she had still dyspnea on exertion. The important new finding was the different $\mathrm{O} 2$ saturation in supine and upright position. In supine position the O2 saturation was $86 \%$ which fell down to $79 \%$ while the patient was upright. Another finding was the resistance of hypoxia to O2 therapy; even 100\% O2 did not increase the level of $\mathrm{O} 2$ saturation. All were in favor of intra-cardiac shunt. This platypnea-orthdeoxia (POS), orthostatic hypoxia, was considered to be related to PFO. She was then, planned to undergo PFO device closure.

The next day, she transferred to the cath-lab. The procedure was guided with intra-cardaic-echocardiography (ICE) under fluoroscopy. The right to left shunt was visible in ICE (Fig. 2; A). Cardiac oximetry was done before device closure. The $\mathrm{O} 2$ saturation in pulmonary vein was $97 \%$ however significant step-down was seen in the left atrium with $\mathrm{O} 2$ saturation of $88 \%$. In addition balloon closure of PFO resulted in rapid increase of systemic saturation up to $95 \%$. Hence, PFO with significant right to left shunt was responsible for patient's symptoms.

At first, PFO closure device FigullaFlex 23-25 mm (Occlutech GmbH, Jena, Germany) was used but retrieved due to significant residual shunt. Then the Amplatzer ASD occluder (AGA Medical Corp, Golden Valley, Minn) 14, was successfully deployed without any significant residual shunt in contrast injection (Fig 2 ; B). The oximetry after wards showed immediate improvement ( $89 \%$ to $94 \%$ ).

The patient was discharged in good condition afterwards.

\section{Case Discussion}

In present study, we described a middle aged woman with DOE from 8 months ago and echocardiographic finding of ruptured sinus Valsalva to RA and PFO. After, trans-catheter device closure the orthostatic hypoxemia (platypnea) was more evident. Hence, she underwent PFO closure afterwards due to platypneaorthodeoxia syndrome (POS).

The aneurysm of sinus Valsalva may be congenital or acquired after infective endocarditis, atherosclerosis or dissection[4]. This rare lesion, may originate from RCC (65\%-85\%), the non-coronary sinus (10\%-30\%), and, rarely, the left coronary sinus $(1 \%-5 \%)[5]$. This pathology is more often seen in Asian male and is frequently asymptomatic. Cases with un-ruptured aneurysm and symptoms of RV outflow obstruction or cerebrovascular emboli have been reported[6]. Ruptured sinus Valsalva aneurysm (RSVA), however, is related to significant cardiac malfunction. Rupture of RCC to RA causes significant left to right shunt, tricuspid regurgitation, RV failure and finally biventricular heart failure may happen[1]. If being uncorrected it will result in deterioration of heart function, hence, early invasive management is advised. Trans-catheter approach is preferred over open surgery in cases with RSVA.

Symptoms in patients with RSVA are mostly acute or subacute. However, the disease course of our patient was chronic. The one explanation may be the presence of PFO decreases a proportion of left to right shunt. High RA pressure because of massive regurgitated flow from aorta caused right to left shunt and decreased RV inflow. This patient did not experience acute RV failure and the size and function of RV were within normal range.

POS is a rare disorder. Both dyspnea and $\mathrm{O} 2$ saturation deteriorates in upright position compared to supine state[7]. Occurrence of this syndrome requires two things; one, intra-cardiac interatrial septal defect or intra- 
pulmonary shunt and two, a functional component during upright standing; interatrial septum deformation. Atrial septum will be deformed while standing and may allow easy streaming of blood from inferior vena cava[8]. The relation between aortic pathologies and POS has been reported in two previous cases. One, in a patient with thoracic aortic aneurysm and another one after Transcatheter Aortic Valve Implantation[9, 10]. As far as we know this is the first case of POS after trans-catheter device closure of RSVA.

\section{Learning points}

The patient had dyspnea on exertion from 8 months ago, however, during many visits; no one carefully auscultated the heart. Even after COVID-19 infection she did not undergo echocardiography to evaluate the other causes of dyspnea.

After successful RSVA closure, the symptoms of the patient were carefully noticed and POS was diagnosed by basic physical examinations. This syndrome is one of the indications of PFO closure[10].

\section{Conclusion}

RSVA is a serious pathology which should be corrected as soon as possible. Trancatheter procedures are available options. Interatrial septum deformation may happen after RSVA correction and right to left shunt from patent foramen oval may become more significant. POS is an important indication for PFO closure which should be noticed by careful examination.

Funding: No funding sources.

Conflict of interest: The authors declare that they have no conflict of interests.

Informed Consent was obtained from the patient.

Consent for Publication Consent for publication was obtained for individual person's data included in the study.

\section{References}

1. Post, M., et al., Rupture of right coronary sinus of Valsalva aneurysm into right ventricle. Netherlands Heart Journal, 2010.18 (4): p. 209-211.

2. Moustafa, S., et al., Sinus of Valsalva aneurysms - 47 years of a single center experience and systematic overview of published reports. The American journal of cardiology, 2007. 99 (8): p. 1159-1164.

3. Mittal, K., et al., Unruptured Noncoronary Sinus of Valsalva Aneurysm Associated with Atrial Septal Defect (ASD). Journal of clinical and diagnostic research: JCDR, 2015. 9 (8): p. TD01.

4. Thankachen, R., et al., Unruptured aneurysm of the sinus of valsalva: presenting with right ventricular outflow obstruction. Texas Heart Institute Journal, 2003. 30 (2): p. 152.

5. Fishbein, M.C., R. Obma, and W.C. Roberts, Unruptured sinus of Valsalva aneurysm. The American journal of cardiology, 1975.35 (6): p. 918-922.

6. Shahrabani, R.M. and P. Jairaj, Unruptured aneurysm of the sinus of Valsalva: a potential source of cerebrovascular embolism.Heart, 1993. 69 (3): p. 266-267.

7. Guerin, P., et al., Transcatheter closure of patent foramen ovale in patients with platypnea-orthodeoxia: results of a multicentric French registry. Cardiovascular and interventional radiology, 2005.28 (2): p. 164168.

8. Akin, E., et al., The platypnea-orthodeoxia syndrome. Eur Rev Med Pharmacol Sci, 2014. 18 (18): p. 2599-604.

9. Roy, A.K., et al., Platypnea-Orthodeoxia Syndrome after Transcatheter Aortic Valve Implantation. Case reports in cardiology, 2016. 2016. 
10. Mann, J., et al., PATENT FORAMEN OVALE CLOSURE IN A PATIENT WITH PLATYPNEAORTHODEOXIA SYNDROME AND A THORACIC AORTIC ANEURYSM.Journal of the American College of Cardiology, 2019. 73 (9 Supplement 1): p. 2300.

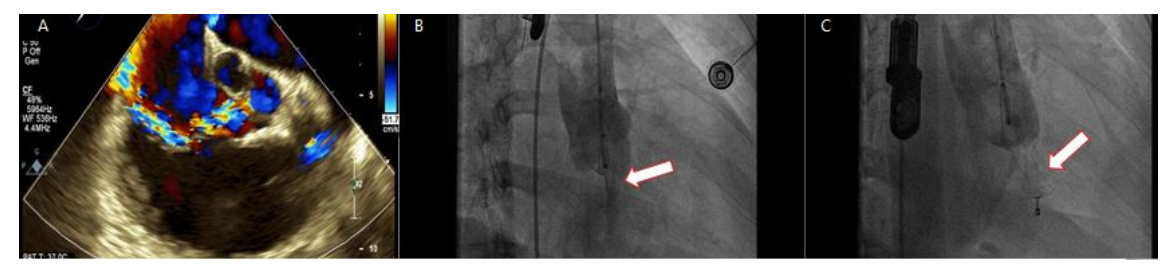

Figure 1. Panel A; Transesophageal echocardiography, rupture of right coronary cusp (RCC) to right atrium (RA), Panel B; cardiac catheterization shows rupture of RCC to RA (white arrow). Panel C; Patent ductus arteriosus occlude is visible in aorta and RA (white arrow).
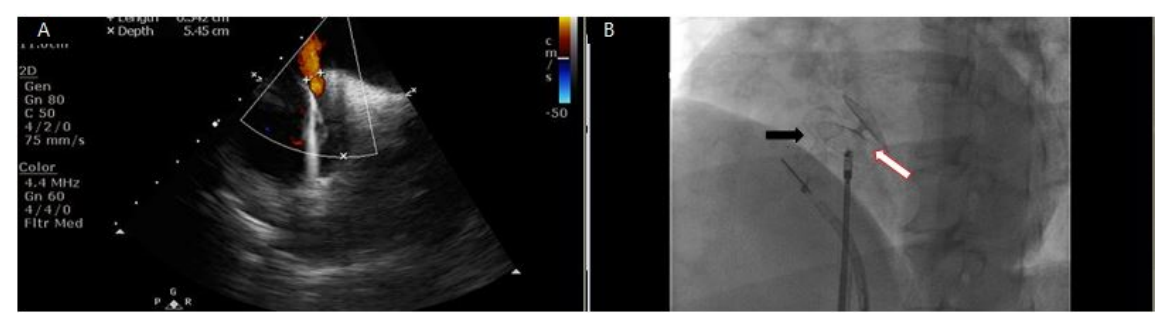

Figure 2, Panel A; Intra-cardiac echocardiography (ICE) shows patent foramen oval (4 mm) with right to left shunt. Panel B; Black arrow is PDA occluder device for ruptured sinus Valsalva and white arrow is an Amplatzer device for PFO closure.

Hosted file

Figure_1_wo_caption.pdf available at https://authorea.com/users/321827/articles/482420orthostatic-hypoxia-after-device-closure-of-ruptured-sinus-valsalva-platypneaorthodeoxia-syndrome

Hosted file

Figure 2 wo caption.pdf available at https://authorea.com/users/321827/articles/482420orthostatic-hypoxia-after-device-closure-of-ruptured-sinus-valsalva-platypnea-

orthodeoxia-syndrome 